treat the thousands of patients with type 1 diabetes. The use of bone marrow as a source of pancreatic $\beta$ cell progenitors has the potential for ex vivo expansion, differentiation, and autologous transplantation. Thus, immunosuppression to prevent rejection could be avoided. Identifying the subpopulation in the bone marrow that gives rise to functional insulin-secreting cells, the mechanism of islet engraftment, as well as the environmental signals that trigger differentiation will be essential for exploiting these cells for the treatment of type 1 and possibly some forms of type 2 diabetes.

1. Bonner-Weir, S., and Sharma, A. 2002. Pancreatic stem cells. J. Pathol. 197:519-526.

2. Swenne, I. 1992. Pancreatic $\beta$-cell growth and diabetes mellitus. Diabetologia. 35:193-201.

3. Fernandes, A., et al. 1997. Differentiation of new insulin-producing cells is induced by injury in adult pancreatic islets. Endocrinology. 138:1750-1762.

4. Pictet, R., and Rutter, W.J. 1972. Development of the embryonic endocrine pancreas. In Handbook of physiology. Volume 1. R.O. Greep, E.B. Astwood D.F. Steiner, N. Freinkel, and S.R. Geiger, editors. American Physiological Society. Washington, DC USA. 25-66

5. Herrera, P.L. 2002. Defining the cell lineages of the islet of langerhans using transgenic mice. Int. J. Dev. Biol. 46:97-103.

6. Finegood, D.T., Scaglia, L., and Bonner-Weir, S. 1995. Dynamics of $\beta$-cell mass in the growing rat pancreas: estimation with a simple mathematical model. Diabetes. 44:249-256.

7. Montanya, E., Nacher, V., Biarnes, M., and Soler J. 2000. Linear correlation between beta cell mass and body weight throughout life in Lewis rats: role of beta cell hyperplasia and hypertrophy. Diabetes. 49:1341-1346.

8. Waguri, M., et al. 1997. Demonstration of two dif ferent processes of beta-cell regeneration in a new diabetic mouse model induced by selective perfusion of alloxan. Diabetes. 46:1281-1290.

9. Gu, G., Dubauskaite, J., and Melton, D.A. 2002 Direct evidence for the pancreatic lineage: NGN3+ cells are islet progenitors and are distinct from duct progenitors. Development. 129:2447-2457.

10. Ianus, A., Holz, G.G., Theise, N.D., and Hussain, M.A. 2003. In vivo derivation of glucose-competent pancreatic endocrine cells from bone marrow without evidence of cell fusion. J. Clin. Invest. 111:843-850. doi:10.1172/JCI200316502.

11. Lagasse, E., et al. 2000. Purified hematopoietic stem cells can differentiate into hepatocytes in vivo. Nat. Med. 6:1229-1234.

12. Theise, N.D., et al. 2000. Derivation of hepatocytes from bone marrow cells in mice afte radiation-induced myeloablation. Hepatology. 31:235-240

13. Wagers, A.J., Sherwood, R.I., Christensen, J.L., and Weissman, I.L. 2002. Little evidence for developmental plasticity of adult hematopoietic stem cells. Science. 297:2256-2259.

14. Jiang, Y., et al. 2002. Pluripotency of mesenchymal stem cells derived from adult marrow. Nature. 418:41-49.

15. Rachdi, L., et al. 2001. Expression of the receptor tyrosine kinase KIT in mature $\beta$-cells and in the pancreas in development. Diabetes. 50:2021-2028.

16. Pipeleers, D.G. 1992 . Heterogeneity in pancreat ic $\beta$-cell population. Diabetes. 41:777-781.

17. Kiekens, R., et al. 1992. Differences in glucose recognition by individual rat pancreatic $\beta$-cells are associated with intercellular differences in glucose-induced biosynthetic activity. J. Clin Invest. 89:117-125.

18. de Vargas, L.M., Sobolewski, J., Siegel, R., and Moss, L.G. 1997. Individual $\beta$-cells within the intact islet differentially respond to glucose. J. Biol. Chem. 272:26573-26577.

19. Shapiro, A.M, et al. 2000. Islet transplantation in seven patients with type 1 diabetes using glucocorticoid-free immunosuppressive regimen. N. Engl. J. Med. 27:230-238.
See the related articles beginning on pages 859 and 869 .

\section{The challenge of molecular medicine: complexity versus Occam's razor}

\author{
Eric A. Sobie, ${ }^{1,2}$ Silvia Guatimosim, ${ }^{1}$ Long-Sheng Song, ${ }^{1}$ \\ and W.J. Lederer ${ }^{1}$
}

${ }^{1}$ Medical Biotechnology Center, University of Maryland Biotechnology Institute, Baltimore, Maryland, USA

${ }^{2}$ Nora Eccles Harrison Cardiovascular Research and Training Institute, University of Utah, Salt Lake City, Utah, USA

J. Clin. Invest. 111:801-803 (2003). doi:10.1172/JCI200318153.

Everything should be made as simple as possible, but not simpler.

\section{-Albert Einstein's comment on Occam's Razor}

Address correspondence to: $\mathrm{W}$. J. Lederer, Medical Biotechnology Center, University of Maryland Biotechnology Institute, $725 \mathrm{~W}$. Lombard Street, Baltimore, Maryland 21201, USA. Phone: (410) 706-8181; Fax: (410) 510-1545 E-mail: lederer@umbi.umd.edu.

Conflict of interest: The authors have declared that no conflict of interest exists. Nonstandard abbreviations used: muscle LIM protein (MLP); phospholamban (PLN); cardiac sarcoplasmic reticulum $\mathrm{Ca}^{2+}$ ATPase (SERCA2a); $\beta$-adrenergic receptor kinase-1 ( $\beta$ ARK1); sarcoplasmic reticulum (SR); mutant myosin binding protein C (MyBP-C $\mathrm{C}_{\mathrm{MUT}}$ ).

\section{Cellular cybernetics}

In the spirit of the recent elucidation of the human genome and the current scientific epoch of bioinformatics, a brute-force therapeutic strategy would theoretically be a perfect remedy for any disease. The essential idea is both comprehensive and unsubtle; the strategy only requires that the therapy fix what was broken. While that sounds simple and possible in this era of rapidly advancing medicine, proteomics, genomics, and targeted pharmacology, it is not. The problem is that the level of complexity of most diseases is great (see Figure 1) and our present knowledge of physiology and pathology is inadequate to undertake such comprehensive repair. Indeed, even nominally simple diseases appear to rapidly develop complexities beyond our current grasp. In the absence of such complete knowledge, current endeavors in molecular medicine are guided by Occam's razor, or the idea that the simplest therapy for the animal model is likely to be effective in treating human disease. This approach is very much in the engineering tradition of fixing what the available tools permit and evaluating alternatives based on empirical rather than theoretical considerations. While this is a reasonable approach, the complexities of many 


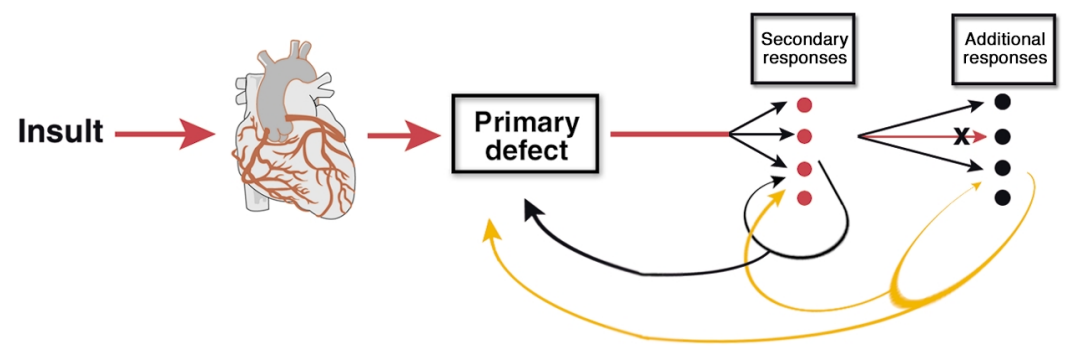

Figure 1

Complexity of signaling in heart failure. Following an insult (e.g., a myocardial infarction), there are many changes in cellular and molecular signals and structure that occur as a primary consequence of that disturbance. Each of the signals and structural changes generated by the sequelae of the insult has many targets, including some earlier in the cascade. These changes in signaling and structure continue to propagate through secondary and later pathways. It is thus possible for therapeutic benefit to arise from interrupting (or augmenting) one of the pathways in the cascade, but it is equally possible for that same change to produce deleterious effects. The left red arrow represents the initial insult. The right red arrow represents a therapeutic intervention The $X$ indicates that the therapeutic intervention may be a functional knockout or an augmentation of the function. Given the complexity of the pathways, the upstream and downstream sense of signaling may not be clear.

diseases make success uncertain, inconsistent, or dependent on special circumstances.

\section{Surprising successes}

As heart failure is a disease that can result from numerous initial causes, and because alterations in many proteins contribute to defective heart function, this disease would on the surface seem to be an unlikely candidate for successful molecular therapy. However, some delightful recent results appeared to defy concerns and deftly overcome the complexity of the disease. In 1999, Minamisawa et al. discovered that a mouse model of heart failure (1) caused by genetic ablation of muscle LIM protein $\left(\mathrm{MLP}^{-/-}\right)$, a structural protein involved in muscle development, could be completely repaired by knocking out another protein, phospholamban (PLN), a regulator of the cardiac sarcoplasmic reticulum $\mathrm{Ca}^{2+}$ ATPase (SERCA2a) (2). Another study found that this same model of heart failure could be significantly repaired by reducing the efficacy of a third protein, the $\beta$-adrenergic receptor kinase- 1 $(\beta \mathrm{ARK} 1)$, by expressing a dominant negative, truncated version of this protein, $\beta$ ARKct $(3,4)$. Similarly encouraging results were found in studies that used gene transfer to overexpress SERCA2a. Because PLN knockout removes the tonic inhibition of SERCA2a by the nonphosphorylated form of PLN, this approach is thought to produce functional effects nearly identical to those of PLN ablation. Overexpression of SERCA2a was shown to improve myocardial performance in the senescent heart (5), to prevent heart failure due to aortic banding (6), and to improve the contractile performance of human heart cells taken from the explanted hearts of patients with heart failure (7).

Mechanistically, it is not obvious why the problems caused by breaking one thing (e.g., $M L P^{-/-}$) can be functionally repaired or prevented by either breaking something else. It is not clear how PLN ablation, overexpression of SERCA2a, or production of a defective protein ( $\beta$ ARKct) reverses the deleterious effects of MLP ablation or other model heart failures. Yet these attempts at molecular rescue of experimental heart failure produced seemingly unambiguous results. One possible explanation for why these different strategies produced similar benefits is that each alters a key signaling pathway that is involved in the transition to heart failure no matter what the nature of the initial insult may be. However, these results also pointed to the complexity of the disease in the sense that changes in either $\beta$-adrenergic signaling or sarcoplasmic reticulum $(\mathrm{SR}) \mathrm{Ca}^{2+}$ regulation could alter the overall phenotype. Despite the difficulty in developing a comprehensive model accounting for all the data, these studies provided hope that a relatively simple solution could overcome the complexity inherent in heart failure. The fact that these genetic manipulations worked in mice suggested that, by Occam's razor, similar interventions might provide effective therapy for humans.

Two papers published in this issue of the JCI $(8,9)$ address the Occam's razor approach to heart failure, i.e., the extent to which relatively simple genetic manipulations can prevent or cure this disease. In contrast to earlier successes in which defective heart function was prevented or reversed, these studies provide a sobering reminder of heart failure's complexity. By examining both animal models and human disease, these papers indicate that the role of PLN in the pathogenesis of heart failure might be more complicated than previously thought.

\section{Failure of PLN ablation to restore function in mouse models}

Song and colleagues (8) examined the ability of PLN ablation to rescue two mouse models of hypertrophic cardiomyopathy caused by either overexpression of Gaq (10) or expression of mutant myosin binding protein $\mathrm{C}$ (MyBP-C MUT $_{\text {) }}$ (11). At the cellular level, crossing these mice with $P L N^{-/}$ mice appeared to rescue the heart failure phenotype in that contraction strength was increased and $\mathrm{Ca}^{2+}$ signaling defects were reversed. However, the molecular therapy of PLN ablation did not reverse the hypertrophy or prevent cardiac dysfunction at the organ level. This outcome emphasizes that PLN ablation may improve cardiac contractile performance by increasing $\mathrm{Ca}^{2+}$ cycling through the $\mathrm{SR}$, but the deletion of this protein does not repair the initial defect that causes the pathology (overexpression of Gaq or mutated MyBP-C). Hence, there is residual pathology.

\section{PLN ablation in humans may be a source of cardiac dysfunction}

Studies on $P L N^{-/-}$mice have indicated that these mice are super-healthy with improved cardiac function relative to control mice and no apparent deleterious effects (12-14). Given the important role played by PLN in regulating cardiac $\mathrm{Ca}^{2+}$ signaling, it seemed odd that such a protein could be removed without any ill effects. Perhaps the paper by Haghighi and colleagues (9) in this issue balances 
that earlier surprise. These investigators examined cardiac function in two families carrying a point mutation in PLN (T116G) that produces a termination codon in place of Leu39. Individuals who are heterozygous for the mutation develop hypertrophy without reduced contractility while those who are homozygous develop dilated cardiomyopathy and heart failure and require early transplantation. Since no PLN protein is detected in the failing hearts, these authors interpret these results as indicating important differences between mice and humans in the effects of the $P L N^{-/-}$ genotype. It is possible, however, that the truncation mutation does not produce a true null and that a truncated protein, for example, is expressed with some consequences.

\section{Overview and cautionary note}

The two new papers discussed here $(8,9)$ challenge the conventional wisdom regarding potential molecular strategies for heart failure. Heart failure is clearly a clinical entity characterized largely by its complexity and presentation rather than by the instigating cause(s). Diverse triggers of heart failure (both sustained and transient), including hypertension, myocardial infarction, contractile filaments proteinopathies, viral myocardiopathies, and diabetes, lead to relatively consistent changes in heart cells. Commonly, the changes include decreased SERCA2a levels, hypophosphorylation of PLN (15), adrenergic activation, hyperphosphorylation of the cardiac ryanodine receptor RyR2 (16), decreased and slowed $\left[\mathrm{Ca}^{2+}\right]_{i}$ transients (17), increased $\mathrm{Na}^{+} / \mathrm{Ca}^{2+}$ exchanger expression (18), decreased excitationcontraction coupling gain (17), unchanged $\mathrm{I}_{\mathrm{Ca}}$ current density, decreased expression of repolarizing potassium currents (19), and prolonged action potentials (20). The relatively common presentation of heart failure suggests that the initiating triggers may activate a common signaling pathway, and the success of molecular therapies aimed at increasing $\mathrm{SR} \mathrm{Ca}^{2+}$ content further suggests that this organelle may be a key player in this common signaling. The two reports featured in this issue $(8,9)$ should now catalyze a reexamination of simple rescue strategies. The reports show that PLN ablation does not rescue or prevent cardiac hypertrophy and failure in some rodent models and that $P L N^{-/-}$per se can underlie serious cardiac dysfunction in humans. However, the animal models used by Song et al. (8) may also contain complexities that are not currently appreciated, and additional genetic factors in the families carrying the PLN null mutation may contribute to the phenotype observed by Haghighi et al. (9). This work also reinforces the importance of continuing the examination of the central role of cellular $\mathrm{Ca}^{2+}$ signaling in the heart failure phenotype. Finally, potential differences between transient and chronic application of $P L N^{-/-}$or SERCA2a overexpression are worth pointing out. It is also possible that, despite the present findings, transient application of these genetic manipulations may become valuable as new inotropes, free of the complications of $\beta$-adrenergic receptor stimulation or protein kinase A activation.

\section{Acknowledgments}

This work has been supported by grants from the National Heart, Lung, and Blood Institute.

1. Arber, S., et al. 1997. MLP-deficient mice exhibit a disruption of cardiac cytoarchitectural organization, dilated cardiomyopathy, and heart failure. Cell. 88:393-403.

2. Minamisawa, S., et al. 1999. Chronic phospholamban-sarcoplasmic reticulum calcium ATPase interaction is the critical calcium cycling defect in dilated cardiomyopathy. Cell. 99:313-322.

3. Rockman, H.A., et al. 1998. Expression of a betaadrenergic receptor kinase inhibitor prevents the development of myocardial failure in gene-targeted mice. Proc. Natl. Acad. Sci. U. S. A. 95:7000-7005.

4. Esposito, G., et al. 2000. Cellular and functional defects in a mouse model of heart failure. Am. J. Physiol. Heart Circ. Physiol. 279:H3101-H3112.
5. Schmidt, U., et al. 2000. Restoration of diastolic function in senescent rat hearts through adenoviral gene transfer of sarcoplasmic reticulum $\mathrm{Ca}(2+)$-ATPase. Circulation. 101:790-796.

6. del Monte, F., et al. 2001. Improvement in survival and cardiac metabolism after gene transfer of sarcoplasmic reticulum $\mathrm{Ca}(2+)$-ATPase in a ra model of heart failure. Circulation. 104:1424-1429.

7. del Monte, F., Harding, S.E., Dec, G.W., Gwathmey, J.K., and Hajjar, R.J. 2002. Targeting phospholamban by gene transfer in human heart failure. Circulation. 105:904-907.

8. Song, Q., et al. 2003. Rescue of cardiomyocyte dysfunction by phospholamban ablation does not prevent ventricular failure in genetic hypertrophy. J. Clin. Invest. 111:859-867. doi:10.1172/ JCI200316738.

9. Haghighi, K., et al. 2003. Human phospholamban null results in lethal dilated cardiomyopathy revealing a critical difference between mouse and human. J. Clin. Invest. 111:869-876. doi:10.1172/ JCI200317892.

10. D’Angelo, D.D., et al. 1997. Transgenic Galphaq overexpression induces cardiac contractile failure in mice. Proc. Natl. Acad. Sci. U. S. A. 94:8121-8126.

11. McConnell, B.K., et al. 1999. Dilated cardiomyopathy in homozygous myosin-binding proteinC mutant mice. J. Clin. Invest. 104:1235-1244.

12. Santana, L.F., Kranias, E.G., and Lederer, W.J. 1997. Calcium sparks and excitation-contraction coupling in phospholamban-deficient mouse ventricular myocytes. J. Physiol. 503:21-29.

13. Wolska, B.M., Stojanovic, M.O., Luo, W., Kranias, E.G., and Solaro, R.J. 1996. Effect of ablation of phospholamban on dynamics of cardiac myocyte contraction and intracellular Ca2+. Am. J. Physiol. 271:C391-C397.

14. Luo, W., et al. 1994. Targeted ablation of the phospholamban gene is associated with markedly enhanced myocardial contractility and loss of beta-agonist stimulation. Circ. Res. 75:401-409.

15. Reiken, S., et al. 2003. Protein kinase A phosphorylation of the cardiac calcium release channel (ryanodine receptor) in normal and failing hearts. Role of phosphatases and response to isoproterenol. J. Biol. Chem. 278:444-453.

16. Marx, S.O., et al. 2000. PKA phosphorylation dissociates FKBP. from the calcium release channel (ryanodine receptor): defective regulation in failing hearts. Cell. 101:365-376.

17. Gomez, A.M., et al. 1997. Defective excitationcontraction coupling in experimental cardiac hypertrophy and heart failure. Science. 276:800-806.

18. Pogwizd, S.M., Schlotthauer, K., Li, L., Yuan, W. and Bers, D.M. 2001. Arrhythmogenesis and contractile dysfunction in heart failure: Roles of sodium-calcium exchange, inward rectifier potassium current, and residual beta-adrenergic responsiveness. Circ. Res. 88:1159-1167.

19. Nabauer, M., Beuckelmann, D.J., and Erdmann, E. 1993. Characteristics of transient outward current in human ventricular myocytes from patients with terminal heart failure. Circ. Res. 73:386-394.

20. Tomaselli, G.F., et al. 1994. Sudden cardiac death in heart failure. The role of abnormal repolarization. Circulation. 90:2534-2539. 